\title{
Erratum: Tighter sum uncertainty relations based on metric-adjusted skew information [Phys. Rev. A 104, 052414 (2021)]
}

\author{
Ruonan Ren ${ }^{\circ}$, Ping Li, Mingfei Ye, and Yongming Li \\ (Received 17 December 2021; published 29 December 2021)
}

DOI: 10.1103/PhysRevA.104.069905

Several errors have been discovered in our original paper.

We have made some changes to Theorems 2 and 3, Corollaries 3-6, Remark 2, and conclusions.

Theorem 2. Let $\Phi_{1}, \Phi_{2}, \ldots, \Phi_{N}$ be arbitrary finite $N(N>2)$ quantum channels, and each channel has Kraus representation $\Phi_{t}(\rho)=\sum_{i=1}^{n} K_{i}^{t} \rho\left(K_{i}^{t}\right)^{\dagger}, t=1,2, \ldots, N$. Then, we have

$$
\sum_{t=1}^{N} I_{\rho}^{c}\left(\Phi_{t}\right) \geqslant \max _{\pi_{t}, \pi_{s} \in S_{n}} \frac{1}{N-2}\left\{\sum_{1 \leqslant t<s \leqslant N} \sum_{i=1}^{n} I_{\rho}^{c}\left(K_{\pi_{t}(i)}^{t}+K_{\pi_{s}(i)}^{s}\right)-\frac{1}{(N-1)^{2}}\left[\sum_{i=1}^{n}\left(\sum_{1 \leqslant t<s \leqslant N} \sqrt{I_{\rho}^{c}\left(K_{\pi_{t}(i)}^{t}+K_{\pi_{s}(i)}^{s}\right)}\right)^{2}\right]\right\},
$$

where $\pi_{t}, \pi_{s} \in S_{n}$ is an arbitrary $n$-element permutation.

Proof. The second inequality of this proof is changed as follows:

$$
\sum_{t=1}^{N} I_{\rho}^{c}\left(K_{\pi_{t}(i)}^{t}\right) \geqslant \frac{1}{N-2}\left[\sum_{1 \leqslant t<s \leqslant N} I_{\rho}^{c}\left(K_{\pi_{t}(i)}^{t}+K_{\pi_{s}(i)}^{s}\right)-\frac{1}{(N-1)^{2}}\left(\sum_{1 \leqslant t<s \leqslant N} \sqrt{I_{\rho}^{c}\left(K_{\pi_{t}(i)}^{t}+K_{\pi_{s}(i)}^{s}\right)}\right)^{2}\right] .
$$

Theorem 3. Let $\Phi_{1}, \Phi_{2}, \ldots, \Phi_{N}$ be arbitrary finite $N(N \geqslant 2)$ quantum channels, and each channel has Kraus representation $\Phi_{t}(\rho)=\sum_{i=1}^{n} K_{i}^{t} \rho\left(K_{i}^{t}\right)^{\dagger}, t=1,2, \ldots, N$. Then, we have

$$
\sum_{t=1}^{N} I_{\rho}^{c}\left(\Phi_{t}\right) \geqslant \max _{\pi_{t}, \pi_{s} \in S_{n}}\left\{\frac{1}{N} \sum_{i=1}^{n} I_{\rho}^{c}\left(\sum_{t=1}^{N} K_{\pi_{t}(i)}^{t}\right)+\frac{2}{N^{2}(N-1)}\left[\sum_{i=1}^{n}\left(\sum_{1 \leqslant t<s \leqslant N} \sqrt{I_{\rho}^{c}\left(K_{\pi_{t}(i)}^{t}-K_{\pi_{s}(i)}^{s}\right)}\right)^{2}\right]\right\},
$$

where $\pi_{t}, \pi_{s} \in S_{n}$ is an arbitrary $n$-element permutation.

Specifically, for arbitrary two quantum channels $\Phi_{1}, \Phi_{2}$ with Kraus representations $\Phi_{1}(\rho)=\sum_{i=1}^{n} K_{i}^{1} \rho\left(K_{i}^{1}\right)^{\dagger}, \Phi_{2}(\rho)=$ $\sum_{i=1}^{n} K_{i}^{2} \rho\left(K_{i}^{2}\right)^{\dagger}$, respectively. Then, we have

$$
I_{\rho}^{c}\left(\Phi_{1}\right)+I_{\rho}^{c}\left(\Phi_{2}\right)=\frac{1}{2}\left[\sum_{i=1}^{n} I_{\rho}^{c}\left(K_{\pi_{1}(i)}^{1}+K_{\pi_{2}(i)}^{2}\right)+\sum_{i=1}^{n} I_{\rho}^{c}\left(K_{\pi_{1}(i)}^{1}-K_{\pi_{2}(i)}^{2}\right)\right],
$$

where $\pi_{1}, \pi_{2} \in S_{n}$ are arbitrary $n$-element permutations, respectively.

Corollary 3. Let $\Phi_{1}, \Phi_{2}, \ldots, \Phi_{N}$ be arbitrary finite $N(N>2)$ quantum channels, and each channel has Kraus representation $\Phi_{t}(\rho)=\sum_{i=1}^{n} K_{i}^{t} \rho\left(K_{i}^{t}\right)^{\dagger}, t=1,2, \ldots, N$. Then, we have

$$
\sum_{t=1}^{N} I_{\rho}^{\alpha}\left(\Phi_{t}\right) \geqslant \max _{\pi_{t}, \pi_{s} \in S_{n}} \frac{1}{N-2}\left\{\sum_{1 \leqslant t<s \leqslant N} \sum_{i=1}^{n} I_{\rho}^{\alpha}\left(K_{\pi_{t}(i)}^{t}+K_{\pi_{s}(i)}^{s}\right)-\frac{1}{(N-1)^{2}}\left[\sum_{i=1}^{n}\left(\sum_{1 \leqslant t<s \leqslant N} \sqrt{I_{\rho}^{\alpha}\left(K_{\pi_{t}(i)}^{t}+K_{\pi_{s}(i)}^{s}\right)}\right)^{2}\right]\right\},
$$

where $\pi_{t}, \pi_{s} \in S_{n}$ is an arbitrary $n$-element permutation.

Corollary 4. Let $\Phi_{1}, \Phi_{2}, \ldots, \Phi_{N}$ be arbitrary finite $N(N \geqslant 2)$ quantum channels, and each channel has Kraus representation $\Phi_{t}(\rho)=\sum_{i=1}^{n} K_{i}^{t} \rho\left(K_{i}^{t}\right)^{\dagger}, t=1,2, \ldots, N$. Then, we have

$$
\sum_{t=1}^{N} I_{\rho}^{\alpha}\left(\Phi_{t}\right) \geqslant \max _{\pi_{t}, \pi_{s} \in S_{n}}\left\{\frac{1}{N} \sum_{i=1}^{n} I_{\rho}^{\alpha}\left(\sum_{t=1}^{N} K_{\pi_{t}(i)}^{t}\right)+\frac{2}{N^{2}(N-1)}\left[\sum_{i=1}^{n}\left(\sum_{1 \leqslant t<s \leqslant N} \sqrt{I_{\rho}^{\alpha}\left(K_{\pi_{t}(i)}^{t}-K_{\pi_{s}(i)}^{s}\right)}\right)^{2}\right]\right\},
$$

where $\pi_{t}, \pi_{s} \in S_{n}$ is an arbitrary $n$-element permutation. 
Specifically, for arbitrary two quantum channels $\Phi_{1}, \Phi_{2}$ with Kraus representations $\Phi_{1}(\rho)=\sum_{i=1}^{n} K_{i}^{1} \rho\left(K_{i}^{1}\right)^{\dagger}, \Phi_{2}(\rho)=$ $\sum_{i=1}^{n} K_{i}^{2} \rho\left(K_{i}^{2}\right)^{\dagger}$, respectively. Then, we have

$$
I_{\rho}^{\alpha}\left(\Phi_{1}\right)+I_{\rho}^{\alpha}\left(\Phi_{2}\right)=\frac{1}{2}\left[\sum_{i=1}^{n} I_{\rho}^{\alpha}\left(K_{\pi_{1}(i)}^{1}+K_{\pi_{2}(i)}^{2}\right)+\sum_{i=1}^{n} I_{\rho}^{\alpha}\left(K_{\pi_{1}(i)}^{1}-K_{\pi_{2}(i)}^{2}\right)\right],
$$

where $\pi_{1}, \pi_{2} \in S_{n}$ are arbitrary $n$-element permutations, respectively.

Corollary 5. Let $\Phi_{1}, \Phi_{2}, \ldots, \Phi_{N}$ be arbitrary finite $N(N>2)$ quantum channels, and each channel has Kraus representation $\Phi_{t}(\rho)=\sum_{i=1}^{n} K_{i}^{t} \rho\left(K_{i}^{t}\right)^{\dagger}, t=1,2, \ldots, N$. Then, we have

$$
\sum_{t=1}^{N} I_{\rho}\left(\Phi_{t}\right) \geqslant \max _{\pi_{t}, \pi_{s} \in S_{n}} \frac{1}{N-2}\left\{\sum_{1 \leqslant t<s \leqslant N} \sum_{i=1}^{n} I_{\rho}\left(K_{\pi_{t}(i)}^{t}+K_{\pi_{s}(i)}^{s}\right)-\frac{1}{(N-1)^{2}}\left[\sum_{i=1}^{n}\left(\sum_{1 \leqslant t<s \leqslant N} \sqrt{I_{\rho}\left(K_{\pi_{t}(i)}^{t}+K_{\pi_{t}(i)}^{s}\right)}\right)^{2}\right]\right\},
$$

where $\pi_{t}, \pi_{s} \in S_{n}$ is an arbitrary $n$-element permutation.

Corollary 6. Let $\Phi_{1}, \Phi_{2}, \ldots, \Phi_{N}$ be arbitrary finite $N(N \geqslant 2)$ quantum channels, and each channel has Kraus representation $\Phi_{t}(\rho)=\sum_{i=1}^{n} K_{i}^{t} \rho\left(K_{i}^{t}\right)^{\dagger}, t=1,2, \ldots, N$. Then, we have

$$
\sum_{t=1}^{N} I_{\rho}\left(\Phi_{t}\right) \geqslant \max _{\pi_{t}, \pi_{s} \in S_{n}}\left\{\frac{1}{N} \sum_{i=1}^{n} I_{\rho}\left(\sum_{t=1}^{N} K_{\pi_{t}(i)}^{t}\right)+\frac{2}{N^{2}(N-1)}\left[\sum_{i=1}^{n}\left(\sum_{1 \leqslant t<s \leqslant N} \sqrt{I_{\rho}\left(K_{\pi_{t}(i)}^{t}-K_{\pi_{s}(i)}^{s}\right)}\right)^{2}\right]\right\}
$$

where $\pi_{t}, \pi_{s} \in S_{n}$ is an arbitrary $n$-element permutation.

Specifically, for arbitrary two quantum channels $\Phi_{1}, \Phi_{2}$ with Kraus representations $\Phi_{1}(\rho)=\sum_{i=1}^{n} K_{i}^{1} \rho\left(K_{i}^{1}\right)^{\dagger}, \Phi_{2}(\rho)=$ $\sum_{i=1}^{n} K_{i}^{2} \rho\left(K_{i}^{2}\right)^{\dagger}$, respectively. Then, we have

$$
I_{\rho}\left(\Phi_{1}\right)+I_{\rho}\left(\Phi_{2}\right)=\frac{1}{2}\left[\sum_{i=1}^{n} I_{\rho}\left(K_{\pi_{1}(i)}^{1}+K_{\pi_{2}(i)}^{2}\right)+\sum_{i=1}^{n} I_{\rho}\left(K_{\pi_{1}(i)}^{1}-K_{\pi_{2}(i)}^{2}\right)\right],
$$

where $\pi_{1}, \pi_{2} \in S_{n}$ are arbitrary $n$-element permutations, respectively.

Remark 2. Notice that the results in Corollaries 5 and 6 completely cover the results of Theorems 2 and 3 in [1]. It can be seen that the number of Kraus operators in each channel are equal. But in fact, they cannot be equal, because if they are not equal, we can solve this problem by filling in zeros.

In Sec. V (CONCLUSIONS AND DISCUSSIONS), the sentence "In addition, the results...channels." is changed to "In addition, the results in this paper covered the results in Ref. [1] about Wigner-Yanase skew information, and we put forward the solution when the number of Kraus operators in each channel are not equal in Sec. IV."

[1] L. M. Zhang, T. Gao, and F. L. Yan, Phys. Lett. A 387, 127029 (2021). 